\title{
Observing Program Calculator for Eclipsing Binary Star Systems
}

\author{
Colby Neal and Michael Braunstein \\ Central Washington University \\ Department of Physics \\ Ellensburg, Washington 98926 USA
}

Received: July 27, $2012 \quad$ Accepted: November 30, 2012

\begin{abstract}
A Microsoft Excel application has been developed to utilize eclipsing binary elements to calculate binary ephemerides that are useful in a program of observing binary systems. Binary star systems consist of two stars orbiting a common center of mass. Consistent monitoring of eclipsing binary stars is important for establishing fundamental astronomical parameters, such as mass and composition of the stellar system. The application that was developed supports effective use of resources in such monitoring applications. The main quantities computed by the application at the time of the eclipse include: local time, Julian date, starting Epoch, altitude of the system, phase of the moon, Local Mean Sidereal Time, and Local Hour Angle. Other quantities computed by the application include current Universal Time (UT), UT/Local Time correction, Julian Date, and current moon phase/percent illuminated. The observing application has been demonstrated to be effective in practical application to obtain differential photometry data for SV Camelopardalis, a system of interest because of variability in its period caused by a third body. The application can be easily modified for use with other highly periodic astronomical systems, for instance transits of extrasolar planets.
\end{abstract}

\section{INTRODUCTION}

The Milky Way Galaxy is estimated to have over 100 billion stars [1]. Roughly half of the stars in the Milky Way are multiple star systems [2]. Multiple star systems can be found using two techniques: visual observation or spectroscopy. Spectroscopy is used in binary systems to exploit the Doppler Effect which causes the spectral lines of the star to be shifted from their rest frame wavelengths [2].

Systems composed of only two stars are commonly known as binary star systems or binary pairs. There are four types of binary star systems: visual, spectroscopic, astrometric, and eclipsing. Visual binaries are systems that can be resolved with the naked eye or through some sort of optical aid (i.e. binoculars or a telescope). A spectroscopic binary is a system with two superimposed, independent, discernible spectra [2]. These systems cannot be resolved by the naked eye or through any optical aid. Spectroscopic binaries are studied through Doppler shifts in the light spectrum of the system. Astrometric binaries consist of systems in which one of the stars is significantly brighter than the other making it difficult or impossible to observe both members directly [2]. The existence of the unseen member may be deduced by observing the proper motion of the system [2]. Eclipsing binaries are systems that have their orbital plane in the line of sight of the observer [2]. This particular orientation allows eclipsing binaries to be studied by observing the system's apparent brightness over a period of time. The apparent brightness of the system will change as one of the stars passes in front of its companion star. Eclipsing binary systems are studied through light curves which are plots of a systems apparent brightness versus time.

Multiple star systems are very significant in astronomy and astrophysics because the only direct way to determine 
the mass of a star is by studying the stars gravitational interaction with other objects [2], which can be very difficult with a single star system. In the case of eclipsing binary systems it has been found to be productive to collect long-term data on the light curves which can, for instance, reveal additional components of the system or other important properties [3-4].

Astronomers use ephemerides, a table of values giving the position of an astronomical object at specific times, to find the location of the object at a specific date and time. The purpose of developing this application was to create a tool to provide a range of useful information for planning observing programs for eclipsing binary systems. Our experience of monitoring eclipsing binaries has resulted in additional features being added to ephemerides that are already readily available, such features proving useful in our own observing program.

We note that the application can be easily modified for use with other highly periodic astronomical systems, for instance transits of extrasolar planets.

\section{OBSERVING APPLICATION}

The observing application uses light elements of eclipsing binary systems obtained from an online accessible source, [5] (though data from other sources can be used as well). This source also provides limited ephemerides for eclipsing binary systems in an online format.

The observing application includes features that the authors have found useful for their eclipsing binary observing program. These include:

\section{Eclipses}

- Local Time and date of the primary and secondary eclipses are displayed for the next 25 periods.

- Eclipses in a user selected time window and above the user selected minimum altitude are highlighted.

Features calculated at time of eclipse

- Epoch

- Julian Date

- Gregorian Date

- Universal Time

- Local Time
- Altitude

- Hour Angle

- Local Mean Sidereal Time

- Percent of Moon illuminated/phase

General Features for current date and time

- Current Julian Date (JD)

- Current Universal Time

- Time Zone Name

- Obtained automatically from users computer

- Whether current date/time is in Daylight Savings Time (DST)

- Date and time of DST transitions

- DST to Standard Time

- Standard time to DST

- Current system phase

- Current Percent of Moon illuminated/phase

System Information

- Sheet 2 titled "System Database" is formatted to accept parameters of the system entered by the user.

- System parameters

- System Name

- $\left(\mathrm{M}_{0}\right)$ "artificial" minimum

- Best fit line set close to Julian Date 2452500, [5].

- Period (days) J2000 Coordinates (RA,DEC)

Eccentricity

Precession of J2000 coordinates are displayed

The application was created in Microsoft Excel because it is a very common and accessible program, many people are accustomed to its layout as well as the general functionality and operation of the program, and Excel does not require an internet connection (as do online ephemeris data).

One key feature of our application is the ability to maintain a database allowing the user to store system parameters for multiple systems. The application makes use of a dropdown menu to select a system from a "database". Using the VLOOKUP function, the application automatically changes the system parameters in the appropriate cells for the selected system in the "Ephemeris Calculator" sheet. The authors have found this "database" feature useful when monitoring multiple systems.

Another feature that was incorporated into our application includes 
the ability to vary the date of starting epoch by use of a dropdown menu. For instance for planning observing sessions at future dates, the application displays times of primary and secondary eclipses that occur starting from the user selected starting date. The application uses the current time from the user's computer and date for starting Epoch as the default setting.

\section{HOW TO USE THE APPLICATION}

The first step in using the application is to enable macros by selecting FILE $\rightarrow$ OPTIONS $\rightarrow$ TRUST CENTER $\rightarrow$ TRUST CENTER SETTINGS $\rightarrow$ MACRO SETTINGS $\rightarrow$ ENABLE ALL MACROS if a dropdown notification does not appear upon opening the application. Cells associated with specific inputs within the application have been highlighted in different colors to make them easier to identify. All of the cells requiring user inputs are highlighted in green. The user inputs include:

- Latitude/Longitude

- Observing time frame

- Local Time Zone Correction

- Selection of Julian date for starting Epoch.

- Minimum system altitude for eclipses

The user's longitude (hours, minutes, seconds) and latitude (degrees, minutes, seconds) are entered in columns D, E, and $F$ in rows 4 and 5 . The user can also alter the observing time frame in column B rows 6 and 7 . The local time zone correction can be changed in cell B12. The minimum system altitude can be changed in cell B8. The minimum system altitude is a limiting parameter which aids in prominently displaying eclipses within the users guidelines. If the user would like to select a different Julian date for the ephemeris to calculate eclipses, a dropdown menu located in cell G:I 1 titled "Use now for starting epoch" can be changed to "Select date for starting epoch". The application will now use the date located in cells G:I 4 as the date for the starting epoch. Once all the user inputs are added into the application the next step is to add systems into the "database".

The application is currently set up to run off a "database" located on sheet two titled "System Database". All of the system parameters are highlighted in blue in the "Ephemeris Calculator" sheet of the workbook. The system input parameters required in the application include:

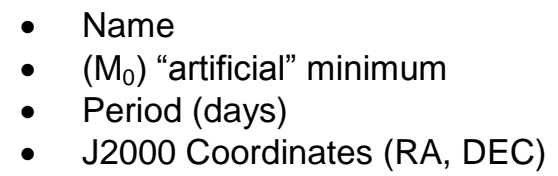

- Note: The application does display the precession of $\mathrm{J} 2000$ coordinates on the "System Database" sheet.

\section{- Eccentricity.}

The application is not set up to handle a system with an eccentricity higher than 0.1 . A system with an eccentricity greater than 0.1 has an elliptical orbit which renders calculations for the time of the secondary eclipse inaccurate. The dropdown menu function in Excel will accommodate up to eight different selections (a limitation imposed by Excel dropdown menu functions), allowing the user to monitor up to eight different systems. In the application "Cutting and Pasting" into the "database" from a separately maintained spreadsheet with the same format can be used to easily use the application for an unlimited number of systems. The "System Name" is in Column A of the "System Database". Once the "System Name" has been entered into the database the Data Validation for Cell $\mathrm{C}: \mathrm{F} 1$ in the "Ephemeris Calculator" sheet needs to be adjusted to incorporate a new entry into the dropdown menu. This is done by selecting the cell C:F 1 then clicking on the "Data" tab followed by "Data Validation" under the "Data Tools" section. Once the "Data Validation" tool opens then a range can be selected in the "System Database" sheet to accommodate a number of systems between one and eight. The system input variables such as $\mathrm{M}_{0}$, Period, Right Ascension, Declination, and eccentricity are entered in columns B through $J$ in the "System Database" sheet. Once all of the system parameters are entered into the "System Database" sheet the application will be able to calculate times of primary and secondary eclipses beginning with the designated starting Epoch.

If the user is interested in observing the system during a particular phase a system phase calculator has been implemented in columns $\mathrm{K}: \mathrm{X}$ rows $7: 12$ in 
the application. The only parameter that the user needs to input is the desired system phase, a value between 0 and 1 , in cell $P: Q$ row $6: 7$

\section{CALCULATIONS}

In order to prevent inadvertent changes to cells that perform calculations, they have been locked. The password for unlocking them is: binary. The application utilizes a number of relationships to calculate the specific time of the eclipses in both user's local time and Universal Time (UT). The eclipsing binary star elements that we used are from data available online, [5]. The binary elements that were obtained online include: system period, starting system epoch, and "artificial" minimum $\left(\mathrm{M}_{0}\right)$ which the Kreiner data [5] selected close to Julian Date 2452500.

The starting Epoch is calculated using two logic functions to determine which Julian Date to use as a starting point. The starting Epoch for calculating eclipses is found according to:

$$
\text { Epoch }_{\text {starting }}=\frac{(\text { Current } J \text { or Selected } J D)-M_{0}}{1 \text { System Period }}-
$$

The Julian Date at the time of the eclipse is found according to:

$$
J D_{\text {eclipse }}=M_{0}+\text { Epoch } * \text { Period } .
$$

The current Julian Date is calculated using:

$$
J D=\text { Date }_{\text {current }}-\frac{U T / L T \text { correction }}{24}+2415018.5
$$

Excel's serial dating system starts as 1 being 12:00AM on January 1, 1900. Therefore, the number 2415018.5 refers to the Julian Date on December 31, 1899 at 12:00AM $[6,7,8]$. The Universal date and time of the eclipse is calculated using:

$$
\text { UT Date } / \text { Time }=J D_{\text {eclipse }}-2415018.5
$$

The user's local time is derived from this calculation by implementing a time correction from the user's time zone [9]. A Visual Basic for Applications (VBA) code, [10], was implemented into the application to call the time zone from the user's computer.
This allows the time zone name to be displayed in cell E13 in the application.

It is the responsibility of the user to enter the Universal Time to Local Time zone correction in cell B12. A table located in column K:L rows $1: 3$ shows Daylight Savings Time to Standard Time transitions and vice versa to aid the user in entering the correct time zone correction.

The altitude of the system is calculated at the time of the eclipse using the observer's latitude, declination, and hour angle of the system in decimal degrees using:

$$
\text { Altitude }=\operatorname{ArcSin}[\operatorname{Sin}(\mathrm{Lat}) * \operatorname{Sin}(\mathrm{Decl})+\operatorname{Cos}(\mathrm{Lat})+\operatorname{Cos}(\mathrm{Decl}) * \operatorname{Cos}(\mathrm{HA})]
$$

The system phase, a number between 0 and 1 that represents the fractional portion of period that has elapsed since the most recent primary eclipse, is calculated using:

$$
\phi=\frac{\text { Starting Epoch }-\left[M_{0}+\left[\text { Rounddown }\left(\frac{J D-M_{0}}{\text { Period }}\right)\right] * \text { Period }\right]}{\text { Period }}
$$

Equation 6 allows the system phase to be calculated at any time using the "Select date for starting epoch". This can be useful to find the time to observe a specific system phase instead of using "use now for starting epoch" (the current date and time).
The application also keeps track of the current moon phase using a VBA code published in the April 1994 digital issue of Sky and Telescope [11]. The code uses:

$$
\begin{gathered}
\alpha=\text { Julian Date }-2451550.1 \\
\beta=\alpha-T_{m} * \operatorname{Int}\left(\alpha / T_{m}\right),
\end{gathered}
$$




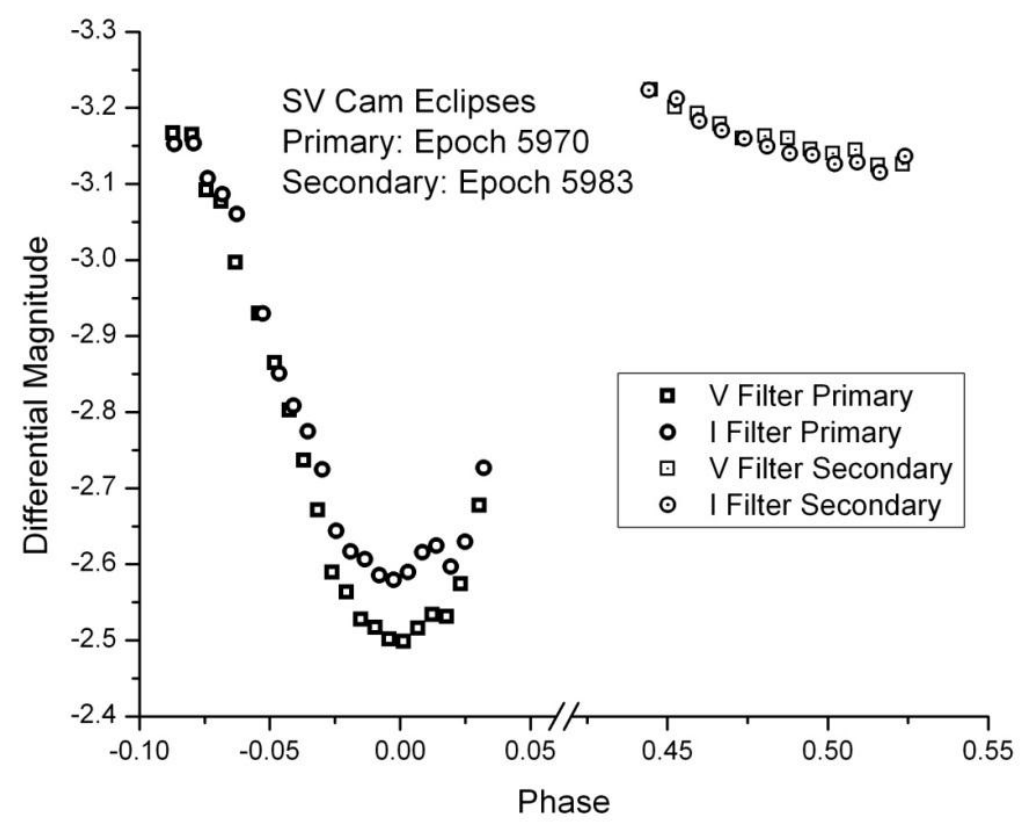

Figure 1. Data taken by students in an advanced upper division physics course using differential photometry of the eclipsing binary system SV Camelopardalis. The data was gathered by employing our application to calculate the times of the primary and secondary eclipses to plan an observing schedule. Data was collected using the Central Washington University 0.3 meter telescope in the Observatory located on top of Lind Hall in Ellensburg, WA.

where $T_{m}=29.83088$ days is the moon's period and $\beta$ will be a quantity between 0 and 29.5309 representing a fraction of the period of the moon phase which can then be used to find the current phase of the moon by:

$$
\text { Phase }=\beta / T_{m}
$$

Using equation 7 we exploited the VBA to implement If-Then statements that also return the name of the Moon phase.

\section{LIMITATIONS}

There are limitations to this application that users should be aware of. The application was designed for use with Excel 2010. One limitation is the long term accuracy of the application. The application is not intended for accurate predictions outside of \pm 50 years from the year 2012 . Some limitations arise from approximations made in calculations. The application does not account for the proper motion of the system and therefore over time the accuracy of equation 5 will be affected. Note that precession will also affect the accuracy of equation 5 because the precession of J2000 coordinates was not incorporated into the altitude calculation. Both of these inaccuracies are very small over the intended 50 year lifespan of the application. One limitation involves how the calculation of ephemerides for secondary eclipses was approached. Close binaries tend to circularize their orbits due to tidal interactions between the two stars. [2]. Therefore, it is common for close binaries to have small eccentricities. For systems with (eccentricity $\leq 0.05$ ) we approximated the time of the secondary eclipses as being at phase $=0.5$. For a system with (eccentricity $>0.05$ ) this approximation may not be accurate due both to the eccentricity and apsidal motion. Using Kepler's Equation [12], to model elliptical orbits, we found that 
for eccentricities $<0.05$, the time calculated by this application for the secondary eclipse will be in error by less than $2 \%$ of the period of the system. For systems with (eccentricity $>0.05)$, the application displays " $X X$ " in the place of the secondary eclipse time. This indicates that the algorithm used to calculate secondary eclipse time (at phase 0.5) may not be accurate.

\section{RESULTS \& CONCLUSIONS}

This observing application for eclipsing binary star systems has proven to be a useful, effective, and reliable resource in monitoring eclipsing binary systems. The application was initially motivated as a tool to plan observation of light curves for the eclipsing binary system SV Camelopardalis and it was shown to accurately calculate times of its primary and secondary eclipses. Figure 1 represents portions of the light curve which were obtained by students in an advanced upper division Physics course using the computing application to calculate the times of the primary and secondary eclipses and plan an observing schedule. The application has also been tested using a variety of eclipsing binary systems such as $\beta$ Persei, $\beta$ Lyrae, and W Uma, cross checking their predicted eclipses with online data [5].

\section{ACKNOWLEDGEMENTS}

This work was supported in part by the Washington Space Grant Consortium, Award NNX10AK64H.

\section{REFERENCES}

1. Bennett, J., Donahue, M., Schneider, N., \& Voit, M. (2009). The Essential Cosmic Perspective. (Fifth ed., pp. 3-4). San Francisco: Pearson Education.

2. Ostlie, D. A., \& Carroll, B. (2006). An Introduction to Modern Stellar Astrophysics. (Second ed., pp. 180-183,
187, 653). Pearson Education.

3. Mullaney, J. (2005). Double and multiple stars and how to observe them. (First ed., pp. 17-19). Singapore: Springer.

4. Percy, J. R. (2007). Understanding variable stars. (First ed., pp. 111-118). New York: Cambridge University Press.

5. Kreiner, J. M. (2004, October 25, 2011). Up-to-date linear elements of eclipsing binaries. Retrieved October 25, 2011, http://www.as.up.krakow.pl/ephem/

6. Fliefel, H. F., \& Van Flandern, T. C. (1968). Letters to the editor: A machine algorithm for processing calendar dates. Communications of the Association of Computing Machines, (11), 657.

7. Seidelmann, K. (1992). Converting between Julian calendar date and Julian date number. In Explanatory Supplement to the Astronomical Almanac (pp. 600-606). Washington D.C.: University Science Books

8. Weisstein, Eric W. (2007). Julian date. Retrieved November 15, 2011, http://scienceworld.wolfram.com/astrono $\mathrm{my} / J u$ lianDate.html

9. The Astronomical Almanac For the Year 2012 (pp. B2-B11). (U.S. Government Printing Office, St. Louis [USA], 2011). http://asa.usno.navy.mil/USNO

10. Pearsons, C. (2008, May 6). Time Zones and Daylight Savings Time. Retrieved January 4 2012, http://www.cpearson.com/excel/TimeZo neAndDaylightTime.aspx

11. Sky and Telescope. April 1994: 86-87 Retrieved March, 16, 2012, http://www.skyandtelescope.com/resour ces/software/3304911.html Filename: MOONFX.BAS

12. Marion, J., \& Thornton, S. (1988). Classical dynamics of particles \& systems. (Third ed., pp. 263-265). Orlando: Harcourt Brace Jovanovich.

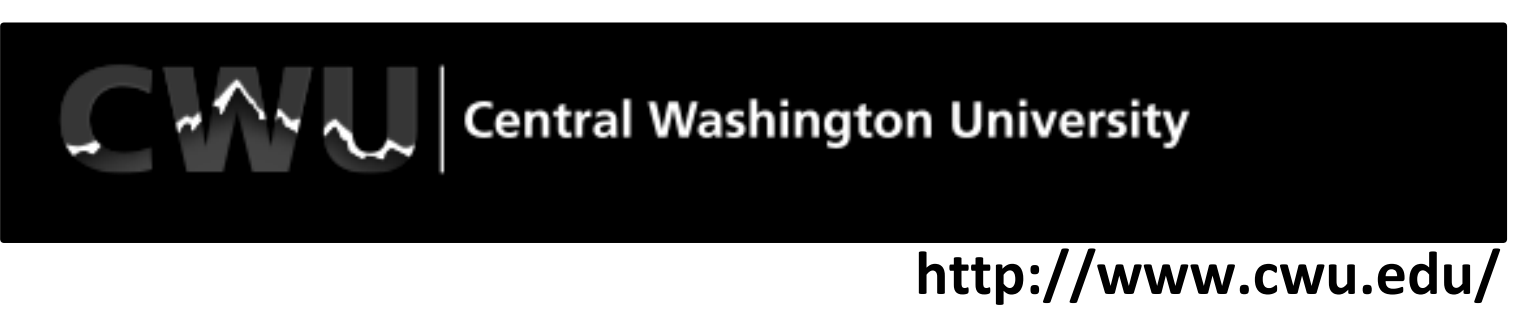

